\title{
A chest wall model based on rib kinematics
}

\author{
Anne-Laure Didier ${ }^{1}$, Pierre-Frédéric Villard ${ }^{2}$, Jacques Saadé ${ }^{1}$, Jean-Michel Moreau ${ }^{1}$, Michaël \\ Beuve $^{1,3}$, Behzad Shariat ${ }^{1}$ \\ ${ }^{1}$ Université de Lyon, Université Lyon 1, Lyon, F-69003, France ; CNRS, LIRIS UMR 5205 \\ ${ }^{2}$ Imperial College London, London W2 1NY, UK \\ ${ }^{3}$ Université de Lyon, Université Lyon 1, Lyon, F-69003, France ; CNRS, IN2P3, IPNL UMR 5822
}

\{anne-laure.didier, jacques.saade@liris.cnrs.fr, p.villard@imperial.ac.uk\}

\begin{abstract}
The success of radiotherapy treatment could be compromised by motion. Lung tumours are particularly concerned by this problem because their positions are subject to breathing motion.

To reduce the uncertainty on the position of pulmonary tumours during breathing cycle, we propose to develop a complete thoracic biomechanical model. This model will be monitored through the measurement of external parameters (thorax outer-surface motion, air flow...) and should predict in real-time the location of lung tumour. In this paper, we expose a biomechanical model of the lung environment, based on anatomical and physiological knowledge. The model includes the skin, the ribs, the pleura and the soft tissue between the skin and the ribcage. Motions and deformations are computed with the Finite Element Method.

The ribcage direct kinematics model, permits to compute the skin position from the ribs motion. Conversely, the inverse kinematics provides rib motion and consequently lung motion. It can be computed from the outer-surface motion.

With regards to available clinical data the results are promising. In particular, the average error is lower than the resolution of the CT-scan images used as input data.
\end{abstract}

Keywords--- Finite Element Method, lung motion, rib kinematics.

\section{Introduction}

Lung cancer is widespread over the world. More than 900000 new cases strike men and 330000 new cases strike women each year. The 5-year survival rate is as low as $15 \%$ for this cancer, likely due to tumour motion during radiotherapy or hadrontherapy sessions. Indeed, lung and liver tumours can move and can be deformed due to patient's breathing $[1,2]$. To now, most of the oncologists add safety margins to anticipate organ deformations and displacements: from the clinical target volume (CTV) a larger planning target volume (PTV) is defined [3]. As a major drawback, a larger fraction of healthy tissues is irradiated limiting therefore the strategy of dose escalation. Alternatively, various methods aiming at reducing the respiratory motion effects have been proposed [4]. The most promising method is based on tracking. It consists in tracking and targeting accordingly the irradiation beam to the tumour during the breathing cycle. Obviously, this approach requires a realtime localisation of the tumour. To this aim several strategies are proposed in the literature: (i) the implantation of fiducial markers gives accurate results. However, this method is invasive and the risk of medical complications, such as pneumothorax [3] is significant; (ii) deducing tumour position from a deformable registration applied to a patient's CT scan images is non invasive. However this method requires the assumption of a reproducible breathing cycle. Breathing reproducibility cannot be guaranteed [5] and it depends on the breathing pattern (thoracic or diaphragmatic respiration) and on the tumour localization [6]. For the same reasons, direct determination of tumour position from the measurement of an external parameter (skin motion...) may lead to position miscalculation. Finally, it seems relevant to model the respiratory system and to drive the model by a set of external parameters (thorax outer-surface motion, air flow...) measured directly during each treatment session, to compute the tumour position.

Another requirement, particularly important in the context of hadrontherapy, would be that this model should predict not only the tumour motion but also the motions, the deformations and the density changes of any tissue traversed by the beam.

As reported in Baudet et al. [7] the models based on discrete approaches that are proposed in the literature [8, $9,10]$ suffer from problems of parameterisation limiting the possibility of the integration of patients' physiological parameters. Alternatively, the continuous methods, which consist in defining every mechanical quantity as continuous functions inside the organs, seem adapted to the radiotherapy context. These methods, generally computed by Finite Elements are known to be 
accurate in many contexts [11]. Moreover density changes can be computed at any point of the deformed organs [19]. Surprisingly, few models based on the continuous approaches focus on respiration modelling $[12,13]$. The model that we proposed aims at fulfilling these requirements. It is based on the anatomy and the physiology of the respiratory system to mimic as possible and with a good accuracy the organs dynamics.

\section{Anatomy and Physiology of the respiratory system}

Lungs are the essential respiration organs. The right lung is composed of three lobes (upper, middle, lower) while the left lung consists of only two lobes (upper, lower) due to heart volume. Left lung and right lung are separated by the mediastinum. These passive structures inflate under the muscles action. The increase of thoracic volume by inspiratory muscles action induces lung expansion, leading to internal negative pressure and consequently to inspiration. The contact of the lungs with the rib cage and the diaphragm is maintained by the pleura. The pleura are composed of two membranes (Figure 1): the first referred as to parietal covers the chest wall, the mediastinum and the diaphragm while the second, referred as to visceral, covers the outer surface of the lungs.

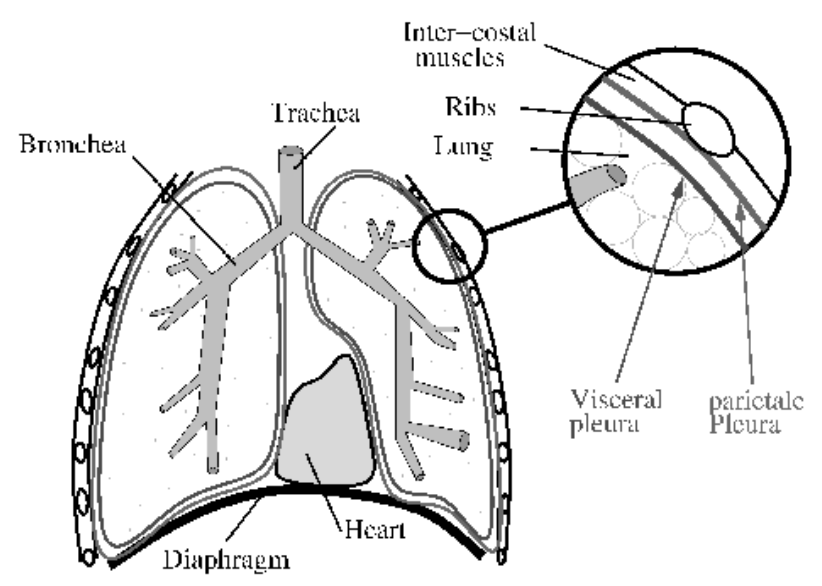

Figure 1. Anatomy of the respiratory system

The space in between the parietal and visceral pleura, known as the pleural space, is filled with an incompressible fluid which lubricates the pleural space and allows the lungs to easily slide against the chest wall during their expansion. Lungs mainly expand by the action of external intercostal muscles (EIM) and the diaphragm. The role of the EIM is relatively important in both quiet and forced repiration. They are inserted into the ribs 2 to 12 and they are responsible for i) the rib elevation and ii) the Posto-Anterior thoracic diameter raise. The diaphragm is a digastric muscle, which separates the thoracic and abdominal cavity. It is composed of two domes: the right dome comes up to the fourth intercostal space whereas the left dome remains below the fifth rib. The diaphragm is constituted of a peripheral part (muscular fibre) and a central tendon. The peripheral part is linked to the whole lower thoracic cavity perimeter and has three major insertions: lumbar, sternum and ribs. During inspiration, the muscular contraction fibres bring down the central tendon. This lowering increases the vertical diameter of the thorax.

Concerning lungs, EIM action induces postoanterior and transversal inflation while the diaphragm action causes vertical motion. Other inspiration muscles (scalenes, sternocleidomastoid...) could be considered, but they play a role only in hyperventilation, a situation that is not relevant in our context.

\section{Our model}

\subsection{General principles}

It appears fundamental to create a lung motion model that integrates the breathing variability to deal with the non-reproducibility of breathing motion. This unpredictability can be explained by the independent action of the diaphragm and the intercostal muscles.

Therefore, our model includes a ribcage model, a diaphragm model and a lung model. The general strategy consists in: i) Deducing the rib cage motion from the thorax outer-surface motion, which will be detected by optical sensors; ii) Deducing the diaphragm motion from other external parameters as inhaled/exhaled air flow or abdominal surface motion iii) Computing the lung deformations induced by the actions of both ribcage and diaphragm.

Notwithstanding, in this paper, we only focus on the dynamic chest wall model. Chest wall modelling aims at establishing a correlation between the ribs and the skin motion on one hand, and between the ribs and lungs motion on the other hand.

We used the finite helical axis method, exposed in a previous study [14] to model the ribs motions from an initial state to an intermediate state. We then obtained for each rib the Euclidean transformation parameters (rotation angle, translation amplitude, axis position and orientation) that have been applied during the simulation.

Skin and Fatty tissues biomechanical parameters are set in accordance with the bibliography [15] and we consider the lung tissues as homogeneous and isotropic, which is a reasonable hypothesis [16].

Our complete model is based on the continuous mechanics laws. It is solved with the finite element method, using Code-Aster software (http://www.codeaster.org/), developed by the French Electricity Board (EDF). We assume that normal breathing can be approximated by a series of quasi-static states and to save computation time we use the small deformation law. The strain tensor is therefore linked to the displacement field by:

$$
\varepsilon_{i, j}=\frac{1}{2}\left(U_{i, j}+U_{j, i}\right)
$$




\subsection{Boundary Conditions}

Our chest wall model boundary conditions are illustrated on Figure 2. Lungs are fixed near the trachea and the pleura behaviour is simulated by applying contact conditions allowing lungs surface to slide against the chest wall (parietal pleura). These contact conditions permit us to model the negative intra-pleural pressure, and the sliding surface represents the pleural fluid. Parietal pleura are directly linked to ribs or fat tissue according to the reality. A particular rigid transformation "Rd" computed with the finite helical axis method is applied to each rib.

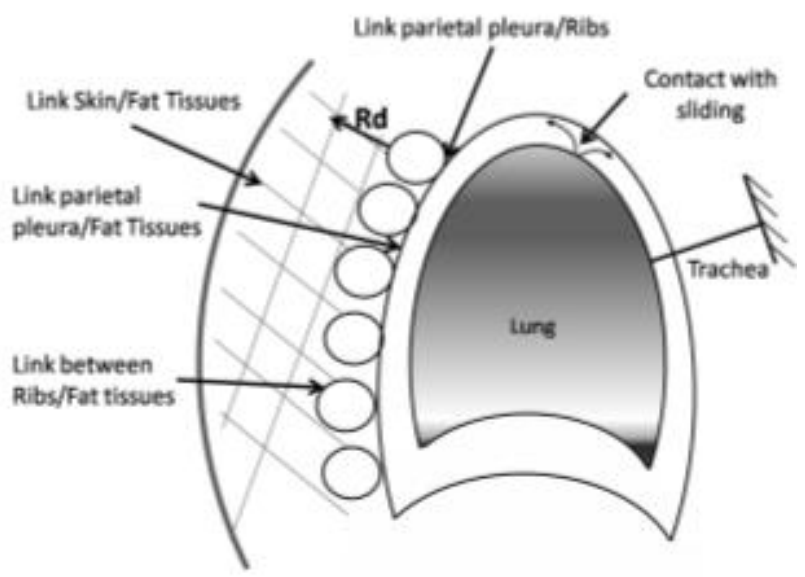

Figure 2. Boundary conditions

\section{Results}

\subsection{CT scan Data}

To create the chest wall model, we have used two sets of two patients' data, that we call Patientl and Patient2. The characteristics are as follows:

Data are available for three breathing levels for Patient1: initial state (end of exhalation), intermediate state and final state (end of inhalation). The CT scan resolution is $0.9375 * 0.9375 * 5 \mathrm{~mm}^{3}$. We have observed a drain on the left anterior part of the thorax.

There are two breathing levels for Patient2: (initial and final state). The CT scan resolution is $0.9375 * 0.9375 * 5 \mathrm{~mm}^{3}$.

\subsection{Mesh generation}

Lungs, skin and ribcage surfaces are extracted from CT scans and are meshed with the marching cube algorithm (Figure3). Then, the volume between ribcage and skin (fatty tissues) as well as the volume inside lungs are meshed with tetrahedrons (http://tetgen.berlios.de/). Our final model is composed by 21000 vertices and 130000 elements (20000 tetrahedrons).
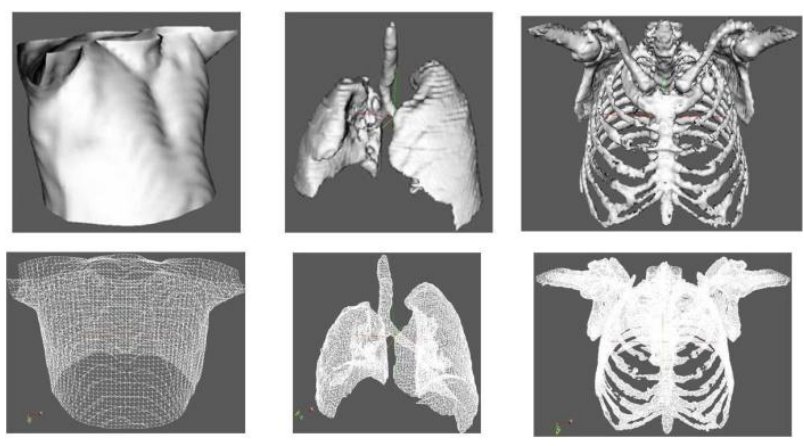

Figure 3. Skin, lungs and thorax. Top: segmentation - bottom: surface meshes

\subsection{Direct Kinematics}

Before simulating the inverse kinematics, we verify the correctness of our direct kinematics model, that permits the computation of chest wall motion: it is important to notice that the direct kinematics model describes the motion of the ribcage, inducing the deformation of fatty tissues and consequently the movement of the skin. This latter deformation can be computed by a Finite Element method.

\subsubsection{Kinematics Simulations}

Several simulations are made to validate our thoracic model. First, we apply the rib displacement corresponding to the transformation computed by finite helical axis method from the end of exhalation position (first CT Scan) to the end of inhalation (Second CT Scan). Then the induced skin motion is computed. For both patients, the distance between the simulated skin motion by our kinematics approach and the segmented skin (from the second CT scan) is estimated. We call this experiment TEST1.

Then, after applying the rib motion, the simulated lungs are compared with the segmented lungs on the final state CT scan. We call this experiment TEST2.

\subsubsection{Kinematics Results}

All the distance maps and the mean errors have been evaluated with an algorithm called MESH, developed by Aspert et al [17]. It allows to estimate the distance between two triangular surface meshes. This method is based on the Hausdorff distance evaluation.

\section{TEST1: distance between simulated and segmented skin}

Figure 4 and Figure 5 show two front views of greyscale distance maps (in $\mathrm{mm}$ ) for both patients. The left side of Figure 4 and Figure 5 illustrates the amplitude of the skin movement between two respiratory states for patient 1 and patient 2 . The right side shows the distance between the simulation of the end of the inhalation state and the reality. Qualitatively, the simulated and segmented surfaces match over the whole thorax. Quantitatively, the average error falls down to 
$2 \mathrm{~mm}$ for patient1 (Figure 4) and to $1 \mathrm{~mm}$ for patient 2 (Figure 5).

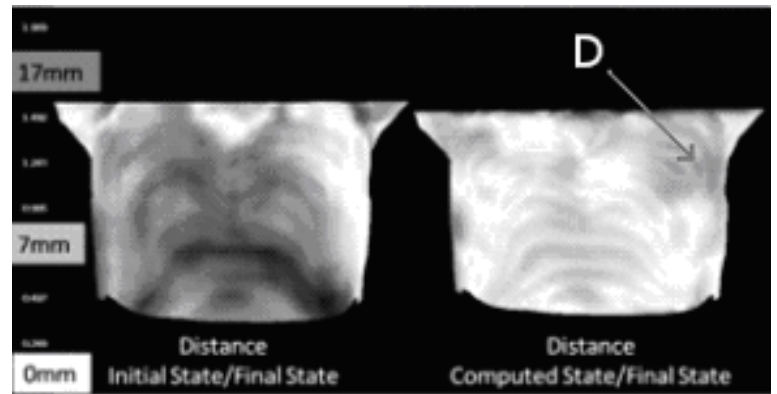

Figure 4. Patient1: left: distance between the initial and final segmented skins - right: distance between computed skin and final segmented skin ( $D$ indicates the drain effect)

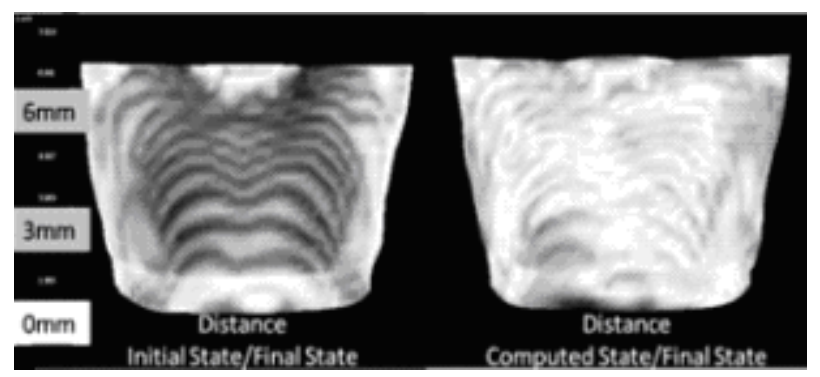

Figure 5. Patient2: left: distance between the initial and final segmented skins - right: distance between computed skin and final segmented skin

\section{TEST2: distance between simulated and segmented lungs}

The results are illustrated on Figure 6 and Figure 7. As previously, the left figure is only to show that there is a real lung motion between the both states measured on CT scan. To evaluate the distance between the computed and segmented lungs, the lower parts of the lungs (all parts beneath the heart's level) are disregarded in order to keep only the lung part which is not influenced by the diaphragm. The mean errors are approximately $5 \mathrm{~mm}$ for Patient 1 and $2 \mathrm{~mm}$ for Patient 2 on the whole lungs surface.

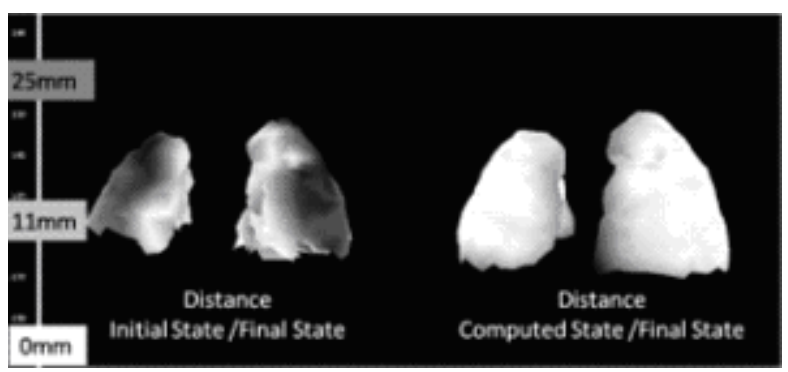

Figure 6. Patient1: distance maps -left: distance between the initial and final segmented lungs right: distance between computed lungs and final segmented lungs

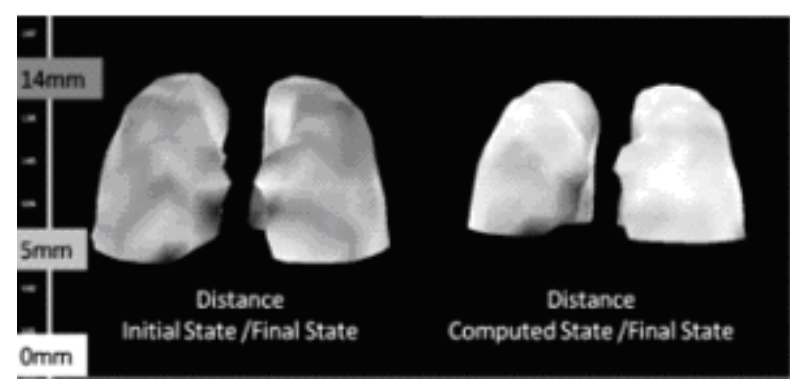

Figure 7. Patient2: distance maps -left: distance between the initial and final segmented lungs right: distance between computed lungs and final segmented lungs

\subsubsection{Kinematics Analysis}

TEST1: distance between simulated and segmented skin

Considering the resolution of the CT scan used as input, the average error between simulated and segmented skin is quite satisfactory. Looking through the results in more details, one can observe for patient 1 an important error on the upper right part of the figure. As previously stated, this is due to the presence of a drain at this location: We can assume that this syringe influences the fat tissues mechanical behaviour. According to the CT scan, the ribs should normally move in this part, but for this patient, the drain induces a non-linearity in the fat tissues that is not introduced in our simulation. We can conclude that except for this artefact the model is demonstrated to be accurate and relevant.

TEST2: distance between simulated and segmented lungs

The mean errors are quite important but if we focus on the local errors on the right of Figures 6 and 7, one can observe that the error is smaller near the rib surface. The mean error increase is caused by the important error near the mediastinum. Indeed the distance map indicates an approximative error of $9 \mathrm{~mm}$ in this part for both patients. It can be caused by the fact that the diaphragm action is not being modelled yet. This part of the lung is influenced by the diaphragm action. However, these results seem to be very promising concerning the link between the rib cage and the lung surface. It demonstrates our direct kinematics model behaviour.

If the computation of direct kinematics is straightforward, the inverse problem is more complicated in our context: knowing the skin motion, the rib mouvement should be deduced.

\subsection{Inverse Kinematics}

\subsubsection{Inverse Kinematics Simulations}

CT scans captured at the initial and at the intermediate state of breathing are used to study the inverse kinematics feasibility. Therefore only Patient1 data could be used. Rib transformation parameters were 
computed using the finite helical axis method with segmented rib cages obtained from CT scans measured at initial and final state of breathing. Using a linear interpolation, the transformation parameters (rotation and translation) are changed as long as the computed skin does not match with the segmented CT-Scan skin at an intermediate state of breathing.

When the distance between these two skins is minimal (approximately equal to the mean error obtained in direct kinematics), we stop modifying the rib transformation parameters and the rib positions obtained are compared with the segmented rib cage from the CT scan taken at an intermediate state of breathing (we call this experiment TEST3). To simplify this procedure while keeping the anatomical reality, we chose to distinguish two rib-motion groups with the same interpolation parameter: the four upper ribs and the last lower ribs.

\subsubsection{Inverse Kinematics Results}

\section{TEST 3: Inverse kinematics}

The right side of Figure 8 illustrates the distance between the intermediate segmented skin and the computed skin. The mean error is approximately $2 \mathrm{~mm}$. The ribs interpolation parameters are then fixed.

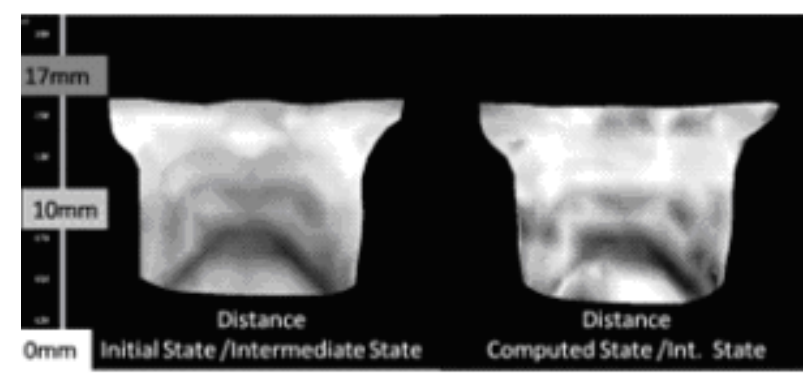

Figure 8. Patient1: distance maps -left: distance between the initial and intermediate segmented skins - right: distance between computed skin and intermediate segmented skin

Two sets of rib interpolation parameters are found; When the rib interpolation parameters are found, the computed skin is closed to the segmented skin, and Figure 9 shows that the distance between the determined rib positions (black points) and the segmented ribs (grey mesh) on the intermediate CT scans is small. The mean error has been estimated by evaluating the distance between reference points on the segmented ribs to the computed ribs. This error is approximately $3 \mathrm{~mm}$.

\subsubsection{Inverse Kinematics Analysis}

\section{TEST 3: Inverse kinematics}

We find two different rib motion groups (upper and lower ribs). Our results are consistent with the conclusions drawn by Ratnovsky et al [18]. They noted that the diaphragm work decreases as breathing effort increases while the work of intercostal muscles increases with breathing effort. After simulation, the computed and the segmented ribcage are very close to each other, especially on the left hand side of Figure 9. These results show that it is reasonable and interesting to build an inverse kinematics model.
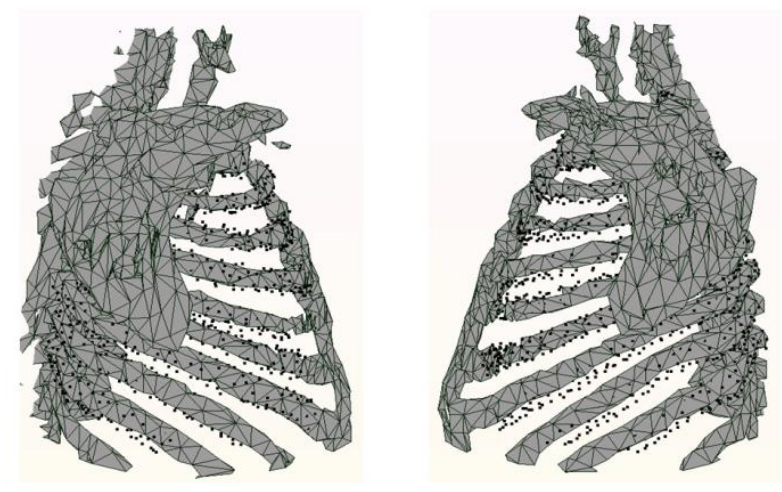

Figure 9 Patient1: 2 thorax sagittal views - grey mesh: thorax segmented on the CT scan at an intermediate state of breathing - black points: computed rib cage by inverse kinematics

\section{Conclusions}

It is essential to predict the lung tumour motion in order to improve radiotherapy or hadrontherapy treatments. Due to the breathing non reproducibility, using a biomechanical model to predict this motion is better than using a model based only on medical imaging. In this study, a relevant lung environment model is presented. Our model allows us to correlate the thoracic outer surface and lung motion with the help of ribs kinematics. In this paper, it has been demonstrated that the inverse kinematics method was conceivable.

In the future we plan to model the diaphragm action to simulate its behaviour. Consequently the real lungs boundary conditions will be completely defined. A relevant motion inside lungs will then be obtained. Finally a tumour should be included in our thoracic model and the inverse kinematics method will be established. The ultimate aim will be to find the rib and diaphragm positions from the thoracic motion obtained by an external sensor and other external parameters like air flow.

\section{Acknowledgments}

We thank the French league against cancer for their financial support and all our partners: Léon Bérard Centre and ETOILE project (Espace de Traitements Oncologique par Ions Légers) for their support.

\section{References}

[1]. Mori S., Endo M., Komatsu S., Yashiro T., Kandatsu S., Baba M. Four-dimensional measurement of lung 
tumor displacement using 256-multi-slice Ct-scanner. Lung Cancer 2007; 56:59-67

[2]. Seppenwoolde Y., Shirato H., Kitamura K., Shimizu S., Van Herk M., Lebesque J.V., Miyasaka K. Precise and real-time measurement of 3D tumour motion in lung due to breathing and heartbeat, measured during radiotherapy. Int. J. Radiation Oncology Biol. Phys. 2002; 53(4), pp. 822-834

[3]. Steve B. jiang Radiotherapy of Mobile Tumors. Seminars in Radiation Oncology 2006; 16:239-248

[4]. Giraud P.,Yorke E., Jiang S., Simon L., Rosenzweig K., Mageras G. Reduction of organ motion effects in IMRT and conformal 3D radiation delivery by using gating and tracking techniques. Cancer Radiothérapie 2006; 10:269-282

[5]. Shirato H., Suzuki K., Sharp G.C., Fujita K., Onimaru R., Fujino M., Kato N., Osaka Y., Kinoshita R., Taguchi H., Onodera S., Miyasaka K. Speed and amplitude of lung tumor motion precisely detected in four-dimensional setup and in real-time tumortracking radiotherapy. Int. J. Radiation Oncology Biol. Phys. 2006; 64(4), pp. 1229-1236

[6]. Handels H.,Werner R.,Schmidt R., Frenzel T., Lu W., Low D., Ehrhardt J. 4D medical image computing and visualization of lung tumor mobility in spatiotemporal CT image data. Int. J. Medical Informatics 2007; 76:433-439

[7]. Baudet V., Villard P.F., , Jaillet F., Beuve M., Shariat B. Towards accurate tumour tracking in lungs. IEEE MediViz, conference on information visualization 2003; pp. 338-343

[8]. Zordan V.B., Celly B., Chiu B., DiLorenzo P.C. Breathe easy : model and control of human respiration for computer animation. Graphical Models 2006; 68: 113:132

[9]. Santhanam P.W., Fidopiastis C.M., Hamza-Lup F.G., Rolland J.P., Imielinska C. Physically-based deformation of high-resolution $3 \mathrm{~d}$ lung models for augmented reality based medical visualization. MICCAI AMI-ARCS 2004; pp. 21-32

[10]. Kaye J., Metaxas D.N.,, Primiano F.P. A 3d virtual environment for modelling mechanical cardiopulmonary interactions. CVR Med, 389-398, 1997

[11].Chi Y., Liang J., Yan D. A material sensitive study on the accuracy of deformable organ registration using linear biomechanical models. Medical Physics 2006; 33(2): 421-433

[12].Brock K.K, Sharpe M.B., Dawson L.A., Kim S.M.; Jaffray D.A. Accuracy of finite element model-based multi-organ image registration. Medical Physics 2005; 32(6):1647-1659

[13].Bettinelli D., Kays C., Bailliart O., Capderou A., Techoueyres P., Lachaud J.L., Vaïda P., Miserocchi G. Effect of gravity and posture on lung mechanics. Journal of applied physiology, 93(6) : 2044-2052, 2002

[14].Didier A.L., Villard P.F., Bayle J.Y., Beuve M., Shariat B. Breathing thorax simulation based on pleura physiology and rib kinematics. IEEE MediVis 2007; pp. 35-40

[15].Handriks F.M. Mechanical behaviour of human skin in vivo - a literature review. Nat.Lab.Unclassified Report 820. Philips Research Laboratories, 2001

[16]. Tai R.C, Lee G.C. Isotropy and homogeneity of lung tissue deformation. J.Biomechanics 1981; 14(4):243252

[17]. Aspert N., Santa-Cruz D., Ebrahimi T. Mesh: measuring errors between surfaces using the
Hausdorff distance. IEEE International conference in multimedia and expo (ICME) 2002; 1: 705-708

[18].Ratnovsky A. and Elad. D. Anatomical model of the human trunk for analysis of respiratory muscles mechanics.Respiratory physiology and neurobiology, 148: 245_262, 2005.

[19].Villard P.F., Beuve M., Shariat B. : An Approach to Convert 4D Geometry into a 4D CT Scan. WSCG (Winter School of Computer Graphics), UNION Agency ed. Plzen (Czech Republic). pp. 163-170. ISBN 80-86943-05. 2006. 